\title{
Forearm and Hand Vein Detection System for an Infrared Image Database
}

\author{
Álvaro Azueto-Ríos, Luis-Enrique Hernández-Gómez, \\ Kevin-Andrés Hernández-Santiago \\ Instituto Politécnico Nacional, BIÓNICA-UPIITA, Mexico City, \\ Mexico \\ aanzuetor@ipn.mx, lehernandezgo@hotmail.com, kevin.andres.hsantiago@gmail.com
}

\begin{abstract}
This paper presents a system that allows defining the vein patterns of a person's forearm and hand. In order to accomplish this, infrared (IR) images of the region of interest were registered. The main goal is to help in vein detection, to aid in procedures like intravenous catheter or venipuncture, in a non-invasive way. In the image acquisition protocol, the anterior and posterior compartments of the left and right forearms of each subject were considered to create an image database each one containing the anthropometric data of the subject. A GUI was developed to allow recording an image by gender, age, weight, high and blood group and to allow two filtering options; the standard histogram equalization or fuzzy equalization. Finally, the user is able to choose and apply one of the two classification methods: one using the Fuzzy C-means algorithm, and the other using a Bayesian probabilistic model.
\end{abstract}

Keywords: Infrared images, vein patterns, GUI, image database, histogram equalization, fuzzy C-means, Bayesian model.

\section{Introduction}

The deoxygenated hemoglobin, which flows within the veins, has the property to absorb the infrared radiation (IR). Crisan \& Tarnovan [1] have measured the absorption pattern, in Figure 1 the obtained data is shown; from this data it is established that maximum absorption range is found in the near infrared spectrum (700-900 nm). This property is exploited by different systems for the vascular detection, either by the transillumination or by reflectometry techniques. The first one consists to expose the forearm to an infrared light source to measure its transparence, however it has the inconvenience that the regions to process need to have the enough thickness to be traversed by the light, restricting its use to the hands and fingers [2-3]. For the reflectometry technique the infrared light is impinged to the exploring surface, which allows to be used regardless of the forearm complexion [4-5]. In both techniques a digital camera is used, which captures the different infrared light intensities and represents them as an image. The main feature of this camera is that it has a filter to allow only the pass of light in the near-infrared range. 
The vascular detection systems have different means to represent and/or display the acquired information. They can be from a simple monitor, where the captured image is displayed, to a graphic user interface (GUI) that allows the user to capture, process and storage images for the creation of a database.

Contrast improvement filters are a topic in the image processing, they are developed to emphasize certain characteristics or accent gray levels off interest. In the last years, studies based in fuzzy logic of this kind of filters had been made. Tizhooch, in [6] has proposed the contrast improvement based in the concept of fuzzy histogram hyperbolization, adding to the classic methods like the adaptive histogram equalization [7].

To improve furthermore the contrast of the acquired images and their later classification, different techniques in the artificial intelligence area, which develops this task, had been proposed (Fuzzy C-Means, FCM and Expectation Maximization, EM). An ulterior phase in the image processing is the segmentation. The image segmentation is a technique that decomposes the images in regions of interest; the pixels, that integrate a region, are classified based on a common feature, like the position hold in the image or its value in gray scale.

In this work, it is presented the development of a GUI for an infrared reflectometry system, which incorporates the processing and classification of objects of interest (the veins) to ease the medical personnel's job to locate the veins in the venipuncture and intravenous catheter procedures. In the next section is detailed the methodology used in this paper.

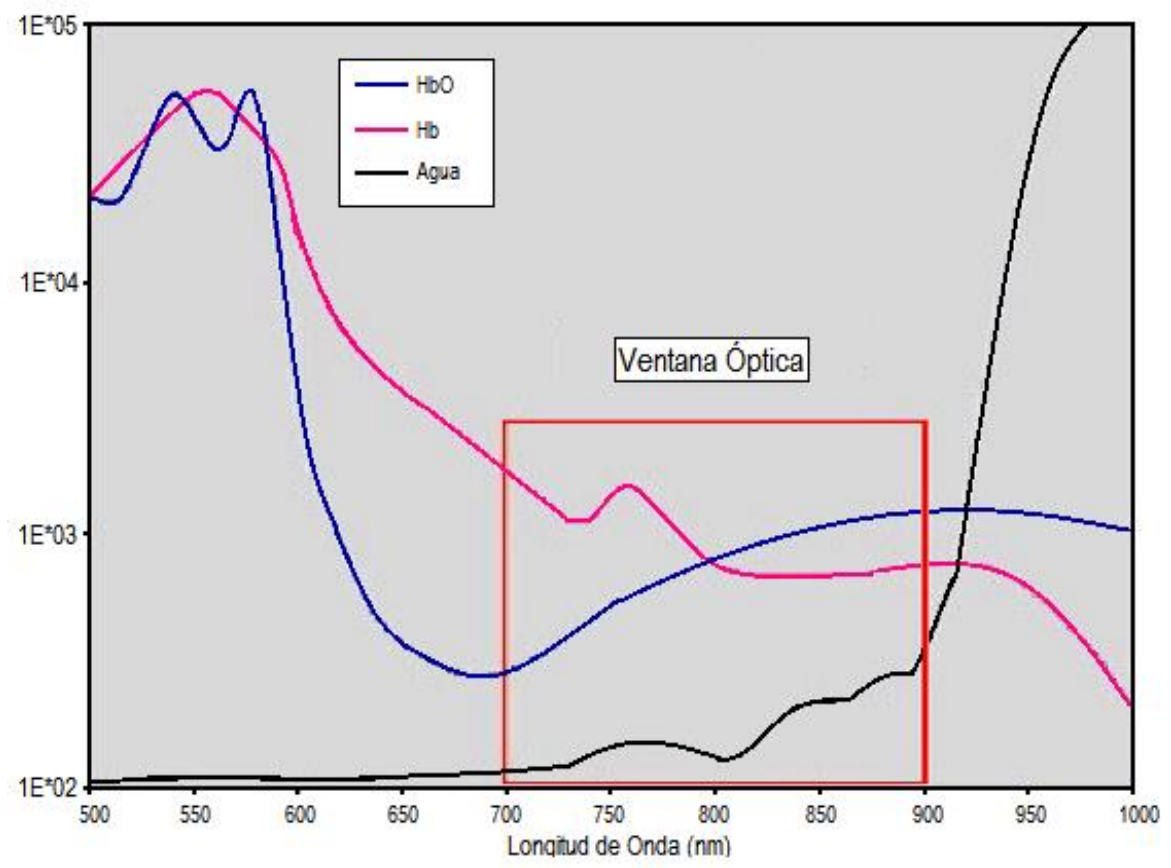

Fig. 1. Optical Window for the IR image acquisition. 


\section{Methodology}

\subsection{IR Image Acquisition System}

The IR image acquisition system is based on a commercial digital camera, "Green Leaf 18-9805", which has an image resolution of $640 \times 480$ pixels and that is able to capture images at 30 frames per second, also it has a light filter that only allows the pass of wave lengths for the visible range.

It is used photographic film that has been thought the photographic process, allowing to be applied as a band pass filter in the wavelength range between $700 \&$ $1200 \mathrm{~nm}$, which appertains to the infrared [8].

The type illumination employed in the vein detection system is reflective. Infrared light is impinged to the exploring surface, capturing the reflected light with the modified camera. The incident light is generated by an array of light emitting diodes (LEDs) that supply wavelength of $80 \mathrm{~nm}$. This array was designed in a circular shape seeking for a uniform illumination upon the exploring region, helping to spotlight the vein distribution. The circular design allows the array to be attached to the camera. In Figure 2a, is shown the modified camera with the LED array.

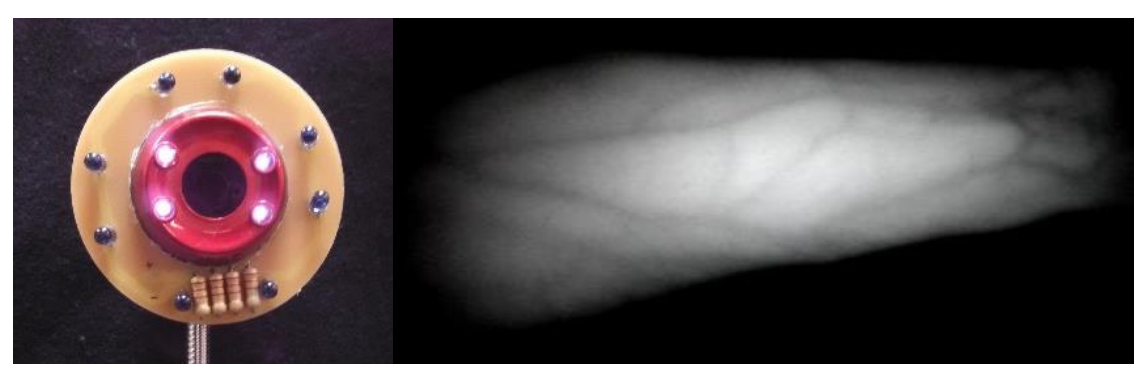

(a)

(b)

Fig. 2. (a) Modified camera attached with the IR LED array (b) Image obtained from the reflective illumination acquisition system.

Figure $2 \mathrm{~b}$ features the resulting image of the acquisition system. It can be noticed that the gray tones that appertain to the veins are obscure. Looking forward to obtain the veins region segmentation, it is applied an ulterior digital processing that allows to enhance the image's contrast.

\subsection{Digital Image Processing}

In image processing, the image's contrast improvement emphasizes certain characteristics, like specific gray scale levels. In this case, the pixels with low numeric values depicts the vein distribution.

This work proposes the utilization of two different methodologies to perform the task of contrast enhancement: the adaptive histogram equalization and the fuzzy hyperbolization. Both methods divide the image in subsections and locally modify the 
pixel values. This local modification has displayed better performance improving the contrast of images with uniform histograms[9].

The adaptive histogram equalization maxes out the image information using the entropy as data scattering metric. This method modifies the gray scale pixel's tonalities, and achieves a redistribution of the histogram [10]. This is made by a function obtained by the pixel's numeric value scattering applied upon a section of the image. Eq. (1) gives the mathematical definition:

$$
s^{\prime}=(1-y)\left((1-x) g_{A}(s)+x g_{B}(s)\right)+y\left((1-x) g_{C}(s)+x g_{D}(s)\right)
$$

where $s$ is the current pixel grayscale level and $s^{\prime}$ is the modified value, $g_{A}, g_{B}, g_{C}$, $g_{D}$ are the histogram mapped value of the restrained pixels in the subsections appertained to each region center A, B, C \& D, at the same time $x$ and $y$ are the normalized distances regarding to the point A.

The second regarded method is the fuzzy histogram hyperbolization which comes from the fact that the human perception of the illumination is not linear [11]. According to that is why the histogram is modified with a logarithmic pattern.

The fuzzy histogram hyperbolization's algorithm is shown in Eq. (2):

$$
g^{\prime}=c\left(\exp \left(\log \left(1+\frac{1}{c}\right) \int_{0}^{g} p(g) d g\right)-1\right)
$$

with $p(g)=\frac{h(g)}{M N}$. Where $\mathrm{M} \& \mathrm{~N}$ are the image dimensions and $h(g)$ is the histogram. To explain furthermore the idea of fuzzy histogram hiperbolization it is necessary to define the membership value of each gray level in respect whit its histogram instead of using its probability. That results in the use of equation (3):

$$
g^{\prime}=\left((L-1)\left(\exp ^{-\mu(g)^{\beta}}-1\right) / e^{-1}-1\right) .
$$

For a membership value $\mu(g)=g-g \min / g \max -g \min$. Where gmax \& gmin are the maximum and minimum, gray intensities in the image.

The next step in the image processing is the segmentation. The image segmentation is the process of pixel grouping based in a common characteristic. To achieve this process as a common characteristic the gray scale intensity levels it is considered. This work uses two different methods to make this grouping, the first one is the socalled Fuzzy C-Means (FCM) and the second one is a Bayesian type classifier, the Expectation Maximization algorithm EM.

The FCM algorithm uses fuzzy set memberships to associate every pixel with at least one cluster. Given an image $X=\left\{x_{i} \in R^{p}, i=1 \ldots n\right\}$, where $\mathrm{n}>0$ is the number of pixels and $\mathrm{p}>0$ is the dimension of the data space of $\mathrm{X}$ let $c \in N, 2 \leq c \leq n$, be the number of clusters in $X$ [12].

Denote $V=\left\{V_{k} \in R^{p}, k=1 . . c\right\}$, as the set of center points of c clusters in the fuzzy partition; $U=\left\{u_{k i} \in[0,1], i=1 \ldots n, k=1 \ldots c\right\}$ as the partition matrix, where $u_{k i}$ is the fuzzy membership degree of the data point $x_{i}$ to the $k^{t h}$ cluster, and 


$$
\sum_{k=1}^{c} U_{k i}=1, \quad i=1 \ldots n .
$$

The clustering problem is to determine the values of $\mathrm{c}$ and $\mathrm{V}$ such that:

$$
(X \mid U, V)=\sum_{i=1}^{n} \sum_{k=1}^{c} u_{k i}\left\|x_{i}-v_{k}\right\| \rightarrow \min ,
$$

where $\|x-y\|$ is the distance between the data points $\mathrm{x}$ and $\mathrm{y}$ in $R^{p}$, defined using Euclidean distance as:

$$
\|x-y\|^{2}=\sum_{i=1}^{p}\left(x^{i}-y^{i}\right)^{2} .
$$

By using fuzzy sets to assign pixels to clusters, FCM allows adjacent clusters to overlap, and therefore provides more information on the relationships among the pixels. In addition, by using a fuzzifier factor, $\mathrm{m}$, in its objective function (7), the clustering model from FCM is more flexible in changing the overlap regions among clusters:

$$
J(X \mid U, V)=\sum_{i=1}^{n} \sum_{k=1}^{c} u_{k i}^{m}\left\|x_{i}-v_{k}\right\| \rightarrow \min ,
$$

where $\mathrm{m}, 1 \leq m \leq \infty$ is the fuzzifier factor.

Equation (7) can be solved using Lagrange multipliers with respect to (4):

$$
\begin{gathered}
V_{k}=\sum_{i=1}^{n} u_{k i}^{m} x_{i} / \sum_{i=1}^{n} u_{k i}^{m}, \\
u_{k i}=\left(\frac{1}{\left\|x_{i}-v_{k}\right\|^{2}}\right)^{\frac{1}{1-m}} / \sum_{j=1}^{c}\left(\frac{1}{\left\|x_{i}-v_{j}\right\|^{2}}\right)^{\frac{1}{1-m}} .
\end{gathered}
$$

To estimate the solution of the system of equations (8) and (9), FCM uses an iteration process. The values of $\mathrm{U}$ are initialized randomly. The values of $\mathrm{V}$ are estimated using (8).

The values of $U$ are then re-estimated using (9) with the new values of V. This process is iterated until convergent where $\exists \varepsilon_{u}>0, T>0 ; \forall t>T$ :

$$
\begin{gathered}
\left\|U_{t+1}-U_{t}\right\|=\max _{k, i}\left\{\left\|u_{k i}(t+1)-u_{k i}(t)\right\|\right\}<\varepsilon_{u}, \\
\mathrm{O}, \exists \varepsilon_{v}>0, T>0 ; \forall t>T, \\
\left\|V_{t+1}-V_{t}\right\|=\max _{k}\left\{\left\|v_{k i}(t+1)-v_{k i}(t)\right\|\right\}<\varepsilon_{v} .
\end{gathered}
$$


The second implemented method comes from a Bayesian classification, based on Expectation Maximization algorithm (EM); it consists in building a model of the distribution of the gray scale levels $\mathrm{Y}$, from an image A, as a mixture of simpler distributions. In this work, it will be make the assumption that each class in the image has a Gaussian distribution, and so the distribution of $\mathrm{Y}$ will be a weighted sum of Gaussian functions [13]. The probability to observe value $y_{i}$ at pixel $i$, knowing that this pixel belongs to class $k$, is:

$$
p\left(y_{i} \mid z_{i}=c_{k}, \mu_{k}, \sigma_{k}\right)=\frac{1}{\sqrt{2 \pi} \sigma_{k}} \exp \left(-\frac{1}{2}\left(\frac{y_{i}-\mu_{k}}{\sigma_{k}}\right)^{2}\right) .
$$

In equation (12), $z_{i}$ is the class of pixel $i$, and $Z$ is the class distribution in the image. Bayes' formula uses this probability in order to find out the probability of pixel $i$ to belong to class $k$, knowing that value $y_{i}$ is observed:

$$
p\left(z_{i}=c_{k} \mid y_{i}, \mu_{k}, \sigma_{k}\right)=\frac{p\left(y_{i} \mid z_{i}=c_{k}, \mu_{k}, \sigma_{k}\right) p\left(z_{i}=c_{k}\right)}{\sum_{k^{\prime}} p\left(y_{i} \mid z_{i}=c_{k^{\prime}}, \mu_{k^{\prime}}, \sigma_{k^{\prime}}\right) p\left(z_{i}=c_{k^{\prime}}\right)} .
$$

In equation (13), $p\left(z_{i}=c_{k}\right)$ is the a priori probability of the $i$-th pixel to belong to class $k$. In order to find out the most likely class for each pixel, we often use a ML (maximum likelihood) approach. Here, it means searching estimates of the Gaussian distribution parameters $\mu k$ and $\sigma k$, denoted by $\hat{\mu}_{k}$ and $\widehat{\sigma}_{k}$. These parameters are intended to maximize a likelihood function:

$$
\begin{gathered}
\hat{\theta}=\arg \max _{\theta} \ell(\theta ; \mathrm{y} ; \mathrm{z}) \\
\text { where } \theta=\left[\mu_{k}, \sigma_{k}\right] k, y=\left[y_{i}\right] i, z=\left[z_{i}\right] i \text {, and } \\
\ell(\theta ; y ; z)=\log p(y, z \mid \theta)=\log \prod_{i} p\left(y i, z i \mid \mu_{k}, \sigma_{k}\right) .
\end{gathered}
$$

Since variable $Z$ is hidden (we are trying to estimate the class $z i$ of each pixel $i$ ), we will use the EM algorithm. This algorithm is divided in two steps:

1. Step E - From an initial value of the Gaussian parameters, estimate with equation (13) the probability that $z_{i}=c_{k}$ for each pixel $i$.

2. Step $\mathbf{M}$ - Update $\hat{\theta}$ by maximizing a modified likelihood function given by:

$$
l^{\prime}(\theta ; y ; z)=p\left(z_{i}=c_{k} \mid y_{i}, \hat{\theta}\right) \sum_{i, k} \log \left(\frac{1}{\sqrt{2 \pi} \sigma_{k}} \exp \left(-\frac{1}{2}\left(\frac{y_{i}-\mu_{k}}{\sigma_{k}}\right)^{2}\right)\right),
$$

where $p\left(z_{i}=c_{k} \mid y_{i}, \hat{\theta}\right)$ have been estimated in step E.

In order to update the Gaussian distribution's parameters, the equation (13) is derived whit respect to $\mu_{k}$ and $\sigma_{k}$, obtaining:

$$
\begin{gathered}
\frac{\partial l^{\prime}}{\partial \mu}=\sum_{i, k} p\left(z_{i}=c_{k} \mid y_{i}, \hat{\theta}\right)\left(\frac{y_{i}-\mu_{k}}{\sigma_{k}^{2}}\right), \\
\frac{\partial l^{\prime}}{\partial \sigma}=\sum_{i, k} p\left(z_{i}=c_{k} \mid y_{i}, \hat{\theta}\right)\left(\frac{\sigma_{k}^{2}-\left(y_{i}-\mu_{k}\right)}{\sigma_{k}^{3}}\right) .
\end{gathered}
$$


By making each derivative equal to zero and solving each resulting equation, we found that:

$$
\begin{gathered}
\mu_{k}=\frac{\sum_{i, k} p\left(z_{i}=c_{k} \mid y_{i} \hat{\theta}\right) y_{i}}{\sum_{i, k} p\left(z_{i}=c_{k} \mid y_{i} \hat{\theta}\right)}, \\
\sigma_{k}^{2}=\frac{\sum_{i, k} p\left(z_{i}=c_{k} \mid y_{i} \hat{\theta}\right)\left(y_{i}-\mu_{k}\right)^{2}}{\sum_{i, k} p\left(z_{i}=c_{k} \mid y_{i}, \hat{\theta}\right)} .
\end{gathered}
$$

\subsection{Graphic User Interface}

To allow the interaction between the user and the infrared image acquisition and processing system it is designed a graphic user interface, it allows creating an image and clinical database of a patient.

The interface has a main window where the relevant clinical data of each patient is inserted:

- Name,

- Gender,

- Age,
- Blood type,

- Weight,

- Size.

Once the data is stored, an option to continue to a new window will be offered, where the current view of the camera, a capture button, and the resolution and zoom options will be displayed. The user will chose the options that allows observing a preliminary vein distribution of the current section of the patient forearm, and the user will be able to capture the current image by a displayed button when it deems so. The captured image will be shown and in a popup window, it will be asked if the user wants to re-take the image, otherwise the interface's next window will be displayed.

In the new window the recorded image and a menu where the options to choose a contrast enhancement filter will be shown. This filter is going to be applied to the original image and the resulted image will be shown in this same window alongside the execution time. The user will have the option to refresh the image or to continue to the next step; in this case, the fourth interface's window will appear.

The fourth window, which one makes the segmentation an classification of the vein system, shows the contrast improved image and a menu with the two available classifiers (EM \& FCM). Once a classifier is chosen, it will be applied, as a result it will be displayed the segmented image alongside the execution time; if the outcome image is not the expected one, a refresh option is available. Finally, the user will be asked if it is wanted to save all the images and data, outcome of the selected processing or if they are going to be discarded and the process will start over. In the case to choose to save the data, the user will have the option to continue with the image acquisition of a new region for the same patient or to input a new one or, if it is the case, to exit the program. 
In Fig. 3 we show the GUI's flow diagram, where its case structures and blocks are displayed.

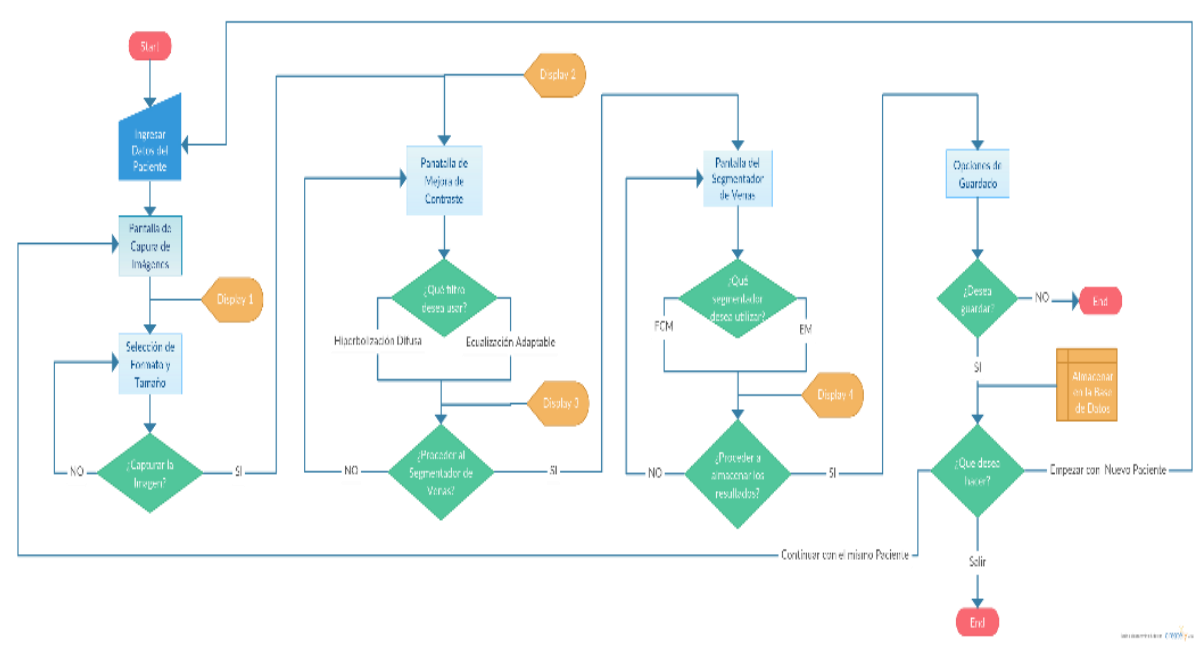

Fig. 3. Vein classification GUI's flow diagram.

\section{Results}

From the proposed methodology a data set of an individual patient from the database is given, see Figure 4.

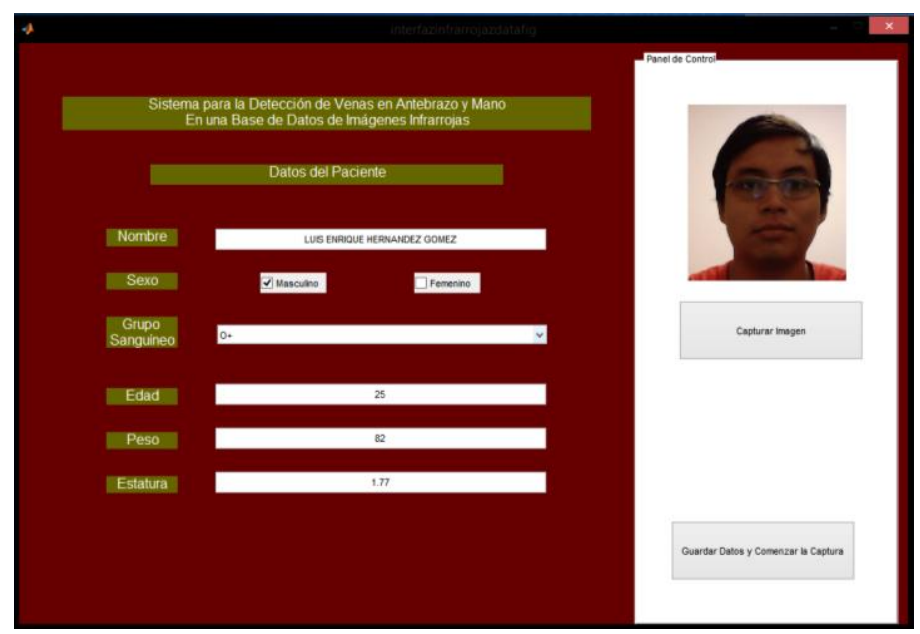

Fig. 4. Main Window where the patient clinical data is inserted.

In Figure 5 the screenshot of the captured image is displayed, it appertains to a forearm's section. The window's main fields are image resolution (640x480), Zoom 
(1x), and 0.5 to brightness and exposure. After selecting the image-capture option, the user will proceed to the contrast-enhancement selection window.

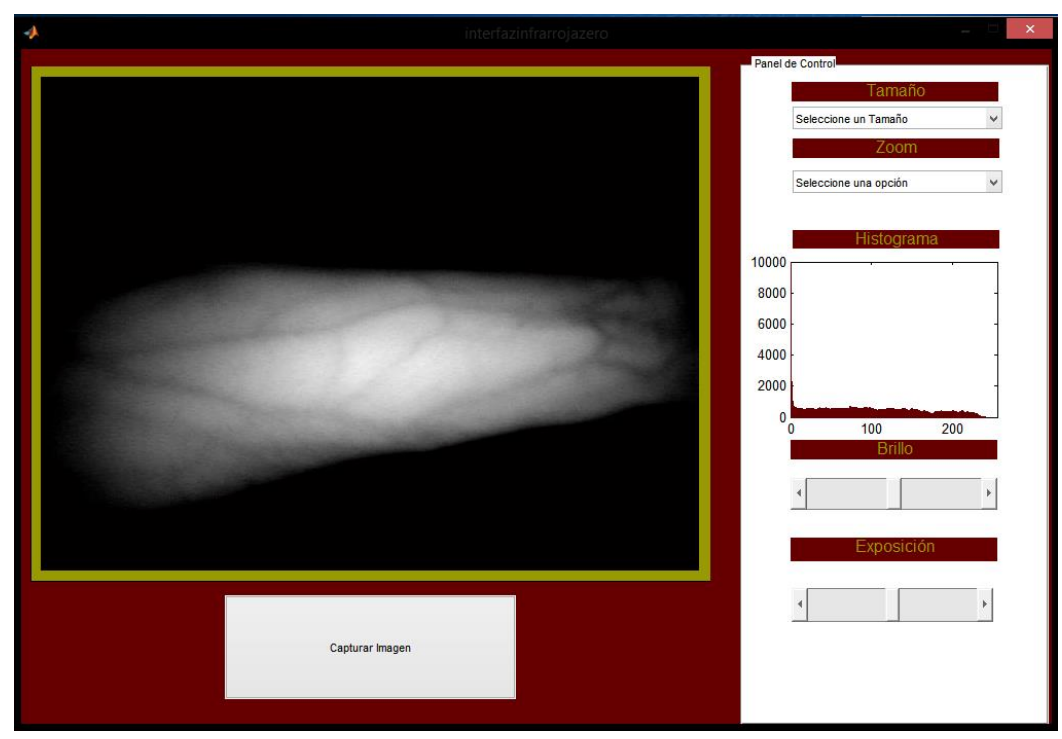

Fig. 5. Image acquisition's control window.

In Figure 6 the contrast improvement options are displayed, having available to methodologies: fuzzy hyperbolization and adaptive histogram equalization. In Figure 6a, the fuzzy hyperbolization is applied, having an execution time of 20.2 seconds. In Figure $6 b$, the adaptive equalization is used with an execution time of 0.12 seconds.

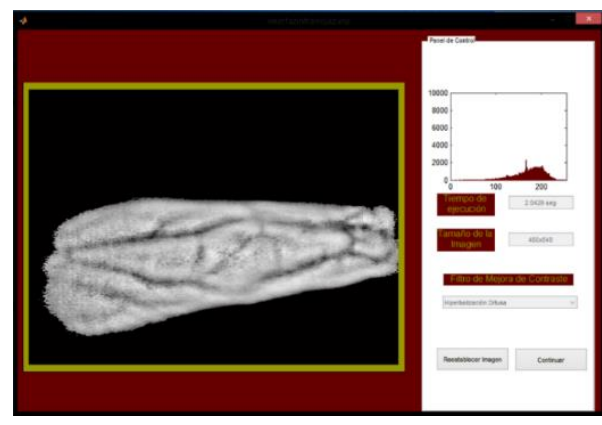

(a)

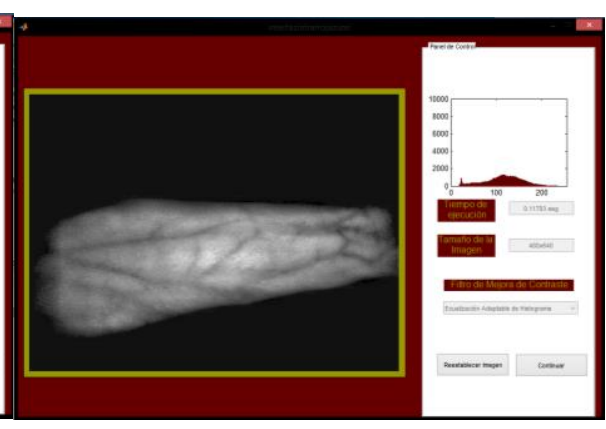

(b)

Fig. 6. Contrast enhancement's selection window (a) Fuzzy hyperbolization outcome (b) Adaptive equalization outcome.

The classification \& segmentation panel is shown in Figure 7, which has two options to make this task. The resulting segmentation by FCM is presented in Figure $7 \mathrm{a}$ and in Figure $7 \mathrm{~b}$ the corresponding to EM. In both options, the result is the 
classification of the vein distribution and the execution times of $0.98 \& 3.73$ seconds, respectively.

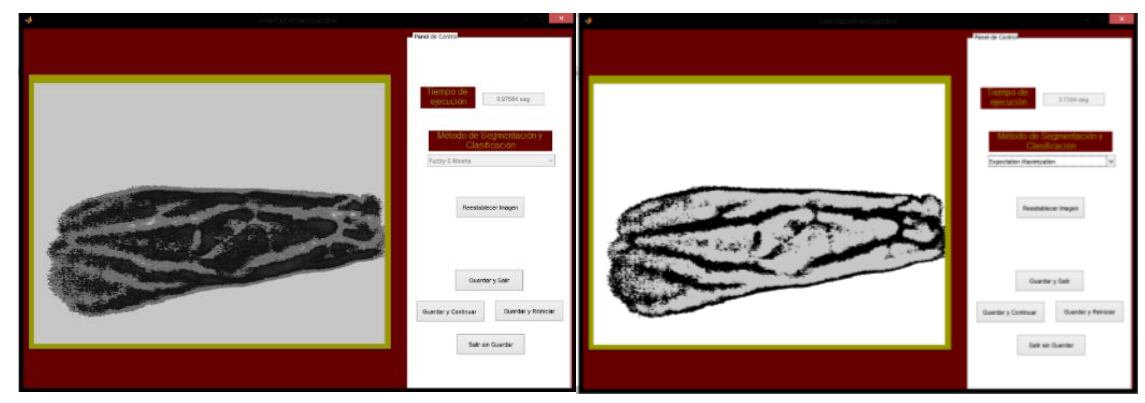

(a)

(b)

Fig. 7. Classifier \& segmentation selection (a) FCM (b) EM outcomes.

Finally, the user will decide if the results, with the selected options \& parameters, are the expected and will have the option to repeat the process for the same patient in a new section. Other options are to input a new patient or to exit the application.

In Table 1, a comparison of the time parameters, obtained by the different algorithm's combinations of the study case is made.

Table 1. Execution times of each algorithm's possible combinations.

\begin{tabular}{lcc}
\hline Contrast Filter & FCM & EM \\
\hline \hline Fuzzy Hyperbolization & $2.12 \mathrm{~s}$ & $5.77 \mathrm{~s}$ \\
Adaptive Equalization & $0.19 \mathrm{~s}$ & $3.85 \mathrm{~s}$ \\
\hline
\end{tabular}

\section{Conclusions}

In this paper, we presented a graphic user interface able to isolate the veins from nearinfrared reflectography images and store them in a database alongside the clinical data of each patient. It had been used the methods of fuzzy hyperbolization \& adaptive equalization to improve the contrast in the original images and two vein segmentation methods the FCM and the EM. The execution times and its easy operation indicate that the system can be used to assist the medical personnel in the venipuncture processes and to generate a database to keep tracking to each patient.

As future perspectives for this development, it will be considered its implementation in a portable continuous response device, which projects the final image upon the processed region. 


\section{References}

1. Crisan, S., Tarnovan, J. G., Criuan, T. E.: A Low Cost Vein Detection System Using Near Infrared Radiation. IEEE, pp. 6-8 (2007)

2. Chakravorty, T., Sonawane, D. N., Sharma, S. D., Patil, T.: Low-cost subcutaneous vein detection system using ARM9 based single board computer. In: ICECT, 3rd Int. Conf. Electron. Comput. Technol., Vol. 2, pp. 339-343 (2011)

3. Limbad, N. V., Parmar, G. D.: Vein Pattern Detection System Using Cost-effective Modified IR Sensitive Webcam. Int. J. Technol. Res. Eng., Vol. 1, No. 9, pp. 975-979 (2014)

4. Shrotri, A., Rethrekar, S. C., Patil, M. H., Bhattacharyya, D., Tai-hoon, K.: Infrared Imaging of $\mathrm{H}$ and Vein Patterns for Biometric Purposes. Vol. 5, pp. 57-66 (2009)

5. Wadhwani, M., Sharma, A. D., Pillai, A., Pisal, N., Bhowmick, M.: Vein Detection System using Infrared Light. Vol. 6, No. 12, pp. 780-786 (2015)

6. Tizhoosh, H. R.: Fuzzy Image Processing. Heidelberg (1997)

7. Zuiderveld, K.: Contrast Limited Adaptative Histogram Equalization. Graph. Gems, Vol. IV, pp. 474-485 (2012)

8. Summerhayes, D.: Digital Infra Red Photography (2004)

9. Kerre, E. E.: Fuzzy Techniques in Image Processing. 1st ed, Gent, Belgium: PhysicaVerlag (2000)

10. Tom V. T., Wolfe, G. J.: Adaptive histogram equalization and its applications. In: Proc. SPIE, Appl. Digit. Image Process. IV, Vol. 354, pp. 204-209 (1982)

11. Frei, W.: Image Enhancement by Histogram Hyperbolization. CGIP, Vol. 6, No. 3, pp. 286-294 (1977)

12. Ross, T. J.: Fuzzy Logic with Engineering Applications (2009)

13. Suetens, P.: Fundamentals of Medical Imaging (2009) 